\title{
X-ray diffraction study of the $\mathrm{Ge}(111) 5 \times 5-\mathrm{Sn}$ and $\mathrm{Ge}(111) 7 \times 7-\mathrm{Sn}$ surfaces
}

\author{
Jan Skov Pedersen, Robert Feidenhans'l, and Mourits Nielsen \\ Physics Department, Risø National Laboratory, DK-4000 Roskilde, Denmark \\ Francois Grey* and Robert L. Johnson \\ Max-Planck-Institute for Solid State Research, D-7000 Stuttgart 80, Federal Republic of Germany
}

(Received 11 May 1988)

\begin{abstract}
We have performed a detailed $x$-ray diffraction study of the structure of the $7 \times 7$ and $5 \times 5$ reconstructions that are observed after deposition of submonolayers of Sn on the Ge(111) surface and subsequent annealing. The structure factors for both reconstructions show considerable similarity with those of $\mathrm{Si}(111) 7 \times 7$. Our analysis shows that the dimer-adatom-stacking-fault model is applicable to the Sn-induced $\mathrm{Ge}(111) 7 \times 7$ and $\mathrm{Ge}(111) 5 \times 5$ structures. The adatoms are identified to be $\mathrm{Sn}$. The structural refinement shows that the atoms in the upper five atomic layers are displaced from their ideal positions. The displacements around the adatoms are similar to the displacements of the $\mathrm{Ge}(111) \sqrt{3} \times \sqrt{3}$-Sn surface. Furthermore, the observed relaxations are in good agreement with the predictions of total-energy calculations. In order to perform a detailed comparison between the experimentally and theoretically determined atomic positions, a series of elastic strain calculations using a Keating model have been carried out. It is demonstrated that this model gives a good description of the atomic relaxations of the surfaces.
\end{abstract}

\section{INTRODUCTION}

Silicon and germanium have similar bulk properties ${ }^{1}$ so it is surprising that the thermodynamically stable reconstructions on the $\mathrm{Ge}(111)$ and $\mathrm{Si}(111)$ surfaces are different. The Ge(111) surface has a reconstruction with a $c(2 \times 8)$ unit cell. ${ }^{2}$ Recent ion scattering and $\mathrm{x}$-ray diffraction studies ${ }^{3}$ have revealed that the structure is described by a simple adatom model which includes atomic relaxations in the upper five atomic layers of the crystal. The Si(111) surface has a $7 \times 7$ unit cell. ${ }^{4}$ The structure of this surface is described by the dimer-adatom-stackingfault (DAS) model deduced by Takayanagi et al. ${ }^{5}$ from transmission electron diffraction measurements. Figure 1 shows the upper layers of the model. The adatoms are in a local $2 \times 2$ arrangement similar to the adatom distribution of the $\mathrm{Ge}(111) c(2 \times 8)$ surface. $^{3}$ The double layer below the adatoms has a stacking fault in half the unit cell. In Fig. 1 it is the part of the unit cell above the short diagonal which has a stacking fault. The regular and faulted parts are connected by rows of dimerized atoms. At the corners of the unit cell, where the rows of dimers meet, they give rise to large corner holes. A notable feature of the model is the extra mirror line along the short diagonal of the unit cell, which is present for the adatoms (shaded circles) and the first double layer (open circles). This line is not a mirror line for the deeper atoms (solid circles), as can be seen in the lower part of Fig. 1. This means that the top layers of the ideal DAS model have $6 \mathrm{~mm}$ symmetry in contrast to the $3 \mathrm{~m}$ symmetry of the bulk layers.

For the Ge(111) surface the fact that the simple adatom model and the DAS model are energetically very similar is demonstrated by the change from $c(2 \times 8)$ to $7 \times 7$ structure under lateral compression ${ }^{6}$ or after deposi- tion of 0.3-0.5 monolayers of Sn and subsequent annealing. ${ }^{7}$ For a Sn coverage of $0.8-1.0$ monolayers a $5 \times 5$ reconstruction is observed, and for $0.5-0.8$ monolayers the surface has a mixture of $5 \times 5$ and $7 \times 7$ structures. The similarity of these structures with the $\operatorname{Si}(111) 7 \times 7$ reconstruction was recognized in 1978 by Ichikawa and Ino, ${ }^{7}$ who observed the Sn-induced structures using reflection high-energy electron diffraction (RHEED). Information on the $\mathrm{Sn}$-induced reconstructions is important

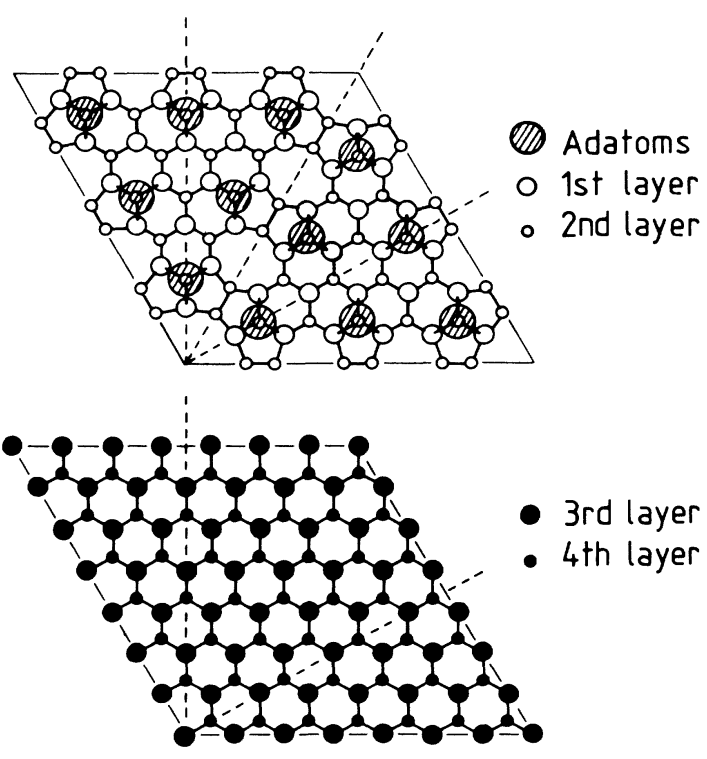

FIG. 1. The top layers of the DAS model. The mirror lines are shown by dashed lines. The first double layer and the adatoms have $6 \mathrm{~mm}$ symmetry. The deeper layers have $3 \mathrm{~m}$ symmetry. 
in order to gain a complete theoretical understanding of the principles underlying the $5 \times 5,7 \times 7$, and $c(2 \times 8)$ reconstructions on the $\mathrm{Si}(111)$ and $\mathrm{Ge}(111)$ surfaces. A structural investigation has to reveal the role of the Sn atoms in the reconstructions. This means that the Sn atoms must be identified and the positions of all atoms in the surface unit cell have to be determined accurately. $\mathrm{X}$-ray scattering experiments are well suited for tackling this problem because the stronger scattering of $x$ rays by $\mathrm{Sn}$ atoms as compared to $\mathrm{Ge}$ atoms enables the location of the Sn atoms to be determined uniquely. Since the number of parameters needed to describe the atomic geometry of these structures is quite large, the intensities of a considerable number of reflections have to be measured. A reliable least-squares optimization of a model requires that the number of nonequivalent reflections measured is between three and five times the number of parameters to be optimized.

Measurements of the in-plane intensity of the fractional-order reflections have been performed on one sample with a $5 \times 5$ reconstruction and two samples with $7 \times 7$ reconstructions. The analysis of these data sets is described below. Part of the work has already been published. ${ }^{8}$ In the present paper the in-plane projected structures are determined, including the positions of the $\mathrm{Sn}$ atoms. The atomic displacements are compared with the results of calculations by the Keating model, ${ }^{9}$ tightbinding calculations, ${ }^{10}$ and experiments on the Si(111) $7 \times 7$ surface. ${ }^{11}$ Finally, the total energy of the surfaces is discussed.

\section{EXPERIMENTAL}

The samples were prepared and characterized at the Flipper II beamline in the Hamburg synchrotron radiation laboratory (HASYLAB). The Ge(111) substrates were cleaned by cycles of sputtering and annealing. Sn was evaporated onto the $\mathrm{Ge}$ substrates at $250-300^{\circ} \mathrm{C}$. The coverages were estimated by a quartz-crystal thickness monitor and core-level photoemission intensities. The estimated coverages are listed in Table I together with other important experimental parameters. The samples with $7 \times 7$ reconstructions are labeled 1 and 2 , respectively. After deposition of the $\mathrm{Sn}$, the samples were

TABLE I. Experimental parameters for the Ge(111)5 $\times 5-\mathrm{Sn}$ and $\mathrm{Ge}(111) 7 \times 7-\mathrm{Sn}$ samples. Estimated coverage $\theta$, angle of incidence $\alpha_{t}$, x-ray wavelength $\lambda$, reproducibility of symmetryequivalent reflections $\epsilon$, the total number of reflections measured, and number of independent reflections, $N^{\text {tot }}$ and $N^{\text {ind }}$, respectively.

\begin{tabular}{lccc}
\hline \hline & & & $7 \times 7$ \\
& $5 \times 5$ & No. 1 & No. 2 \\
\hline$\Theta(\mathrm{ML})$ & & 0.5 & 0.3 \\
$\alpha_{t}(\mathrm{deg})$ & 0.7 & 0.265 & 0.270 \\
$\lambda(\AA)$ & 0.275 & 1.365 & 1.343 \\
$\epsilon$ & 1.344 & 0.10 & 0.05 \\
$N^{\text {tot }}$ & 0.10 & 121 & 360 \\
$N^{\text {ind }}$ & 163 & 90 & 269 \\
\hline \hline
\end{tabular}

annealed at $500^{\circ} \mathrm{C}$ for a few minutes and then cooled slowly to room temperature. The samples were characterized by low-energy electron diffraction (LEED) and photoemission before being transferred to a small portable ultrahigh vacuum chamber for the $\mathrm{x}$-ray diffraction experiment.

During the data collection at the diffractometer at the Wiggler beamline W1 at HASYLAB a standard reflection was measured regularly to check for a possible sample deterioration. Typically the data collection lasted for 3-4 $d$ and no significant decrease of the standard reflection was observed. The in-plane intensities were measured with a grazing angle of incidence scattering geometry.

The structure factor intensities are obtained after correcting the measured integrated intensities for the Lorentz factor and for the active sample area. The reproducibility, $\epsilon$ in Table I, is calculated from the symmetryequivalent reflections as described in Ref. 12 and the uncertainties of the structure factor intensities are calculated from $\epsilon$ and the counting statistics. For the $5 \times 5$ sample, a total of 115 independent reflections with a reproducibility of 0.10 are available. For the best $7 \times 7$ sample (sample 2), which has a reproducibility of $0.05,269$ independent reflections were recorded. The measured structure factor intensities are tabulated in Ref. 12 .

\section{ANALYSIS}

The fractional-order structure factor intensities were used to construct the contour maps of the Patterson (pair correlation) function ${ }^{13}$ shown in Fig. 2 . The peaks in the

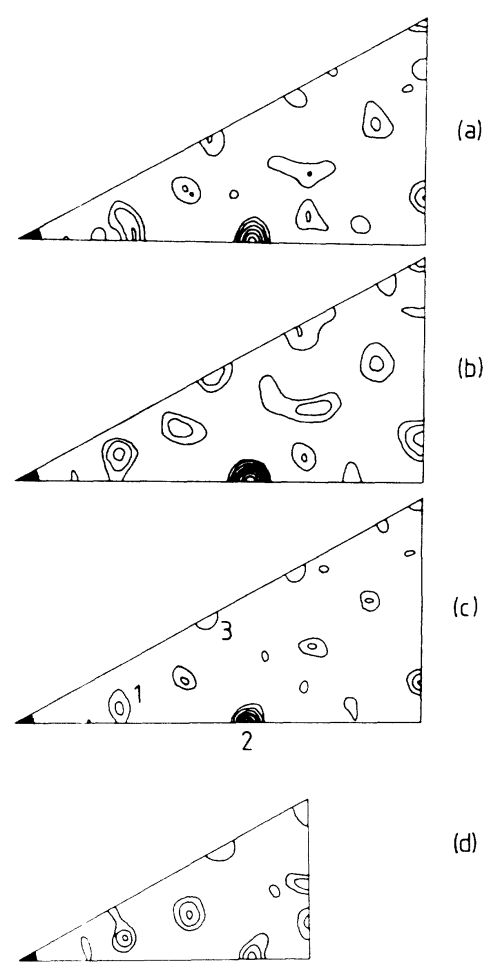

FIG. 2. Plots of the positive contours in the irreducible unit (see Fig. 3) of the Patterson functions. (a) The $\mathrm{Si}(111) 7 \times 7$ surface (after Ref. 11). (b) and (c) The Ge(111)7 $\times 7$-Sn samples 1 and 2, respectively. (d) The $\mathrm{Ge}(111) 5 \times 5$-Sn reconstruction. 
Patterson function correspond to interatomic vectors in the unit cell. The integer-order reflections contain contributions from both the substrate and the surface reconstruction. The systematic omission of these reflections in the sum of the Patterson function leads to negative peaks. However, it has been shown by Bohr et al. ${ }^{14}$ that the positive peaks can still be interpreted as interatomic vectors. Only the positive contours in the irreducible unit (see Fig. 3) have been drawn in Fig. 2. The Patterson function of the $\mathrm{Si}(111) 7 \times 7$ surface is also shown for comparison. ${ }^{11}$ The plots for the $7 \times 7$ structures are quite similar with the sharpest peaks for sample 2, which has the largest number of reflections.

The strongest peaks are at nearly identical positions in all four maps and the overall similarity suggests that the structural models are the same. The similarity of the Patterson functions for the $5 \times 5$ and $7 \times 7$ structures shows that the local atomic arrangement are the same. A detailed analysis of the Patterson function will not be given here $^{5}$ and the $7 \times 7$ DAS model will simply be taken as the starting point for the structure factor analysis. We note, however, that the peak labeled 2 in Fig. 2(c) is the strongest and it corresponds to the adatom-adatom separation, the peak labeled 1 is due to the dimers and the peak labeled 3 has contributions from the stacking fault. The ingredients of the DAS model can be combined to form reconstructions with $(2 n+1) \times(2 n+1)$ unit cells. The structure with $5 \times 5$ and $7 \times 7$ unit cells are shown in Fig. 3. The similarities between the plot of the Patterson function for the $5 \times 5$ and $7 \times 7$ structures demonstrate that the reconstructions have many interatomic vectors in common. Therefore, the $5 \times 5 \mathrm{DAS}$ model is chosen as the starting point for the analysis of the Sn-induced $5 \times 5$ reconstruction.

The independent atoms in the top layers of the $7 \times 7$ and $5 \times 5$ DAS models are shown in Figs. 4(a) and 4(c). The large circles indicate adatoms, medium and small circles indicate first- and second-layer atoms, respectively. The rest of the atoms in the unit cell can be generated by reflections in the bulk mirror lines shown as dashed lines in the figure. The extra mirror line in the top layers of the DAS models is indicated by a dashed-dotted line.

The DAS models with $6 \mathrm{~mm}$ symmetry were the first models that were tested. The model structure factor is given by

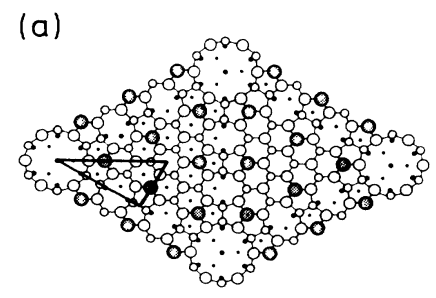

(b)

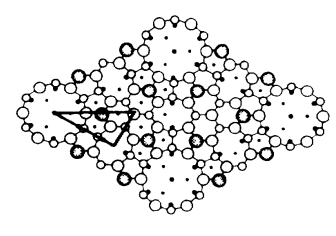

FIG. 3. The DAS model. (a) $7 \times 7$ and (b) $5 \times 5$. The adatoms are shaded and the irreducible unit of the Patterson function is shown by a triangle. (a)

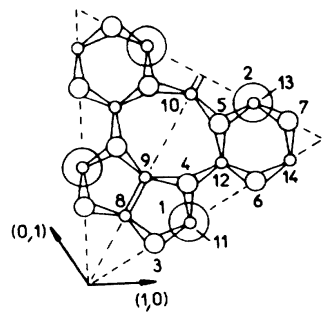

(b)

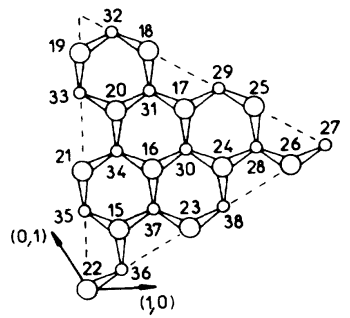

(c)

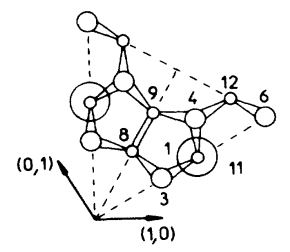

(d)

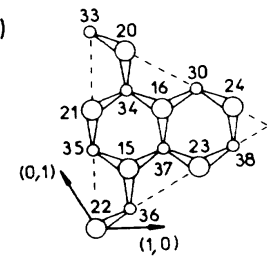

FIG. 4. The atoms in the first two bilayers of the $7 \times 7$ and $5 \times 5$ DAS models. (a) First bilayer and adatoms in the $7 \times 7$ structure. (b) Second bilayer in the $7 \times 7$ structure. (c) First bilayer and adatoms in the $5 \times 5$ structure. (d) Second bilayer in the $5 \times 5$ structure. In the least-squares fits, the upper bilayer and the adatoms were allowed to relax in $6 \mathrm{~mm}$ symmetry and the lower atoms in $3 \mathrm{~m}$ symmetry. For the $7 \times 7$ model the atoms labeled 18 to 26 were fixed and for the $5 \times 5$ model the atoms 20,21, 23, and 24 were fixed.

$$
F_{h k}^{\bmod }=\sum_{p} f_{p}(h, k) e^{-B_{p} Q^{2} /(4 \pi)^{2}} e^{i 2 \pi\left(h x_{p}+k y_{p}\right)},
$$

where the sum is over the atoms in the unit cell. For the $p$ th atom $f_{p}(h, k)$ is the atomic form factor,

$$
e^{-B_{p} Q^{2} /(4 \pi)^{2}}
$$

is the Debye-Waller factor, and $\left(x_{p}, y_{p}\right)$ is the in-plane projected position in units of the basis vectors of the unit cell. In the analysis the agreement between model structure factor intensities $\left|F_{h k}^{\text {mod }}\right|^{2}$ and the experimental structure factor intensities $\left|F_{h k}^{\text {expt }}\right|^{2}$ was evaluated using the least-squares residual:

$$
\chi^{2}=\frac{1}{N-p} \sum_{h k} \frac{\left(\left|F_{h k}^{\text {mod }}\right|^{2}-\left|F_{h k}^{\text {expt }}\right|^{2}\right)^{2}}{\sigma_{h k}^{2}},
$$

where $\sigma_{h k}$ is the standard error on $\left|F_{h k}^{\operatorname{expt}}\right|^{2}, N$ is the number of reflections, and $p$ is the number of parameters to be optimized. As a first step in the analysis of the Ge(111)$\mathrm{Sn}$ reconstructions all atoms were taken to be $\mathrm{Ge}$ with a common isotropic Debye-Waller (DW) factor. Only the positions of the dimer atoms labeled 8, 9, and 10 in Fig. 3 were allowed to vary, and the rest of the atoms were fixed at their ideal positions. For the $\mathrm{Ge}(111) 7 \times 7-\mathrm{Sn}$ data sets 1 and 2 the residuals for the model were, respectively, $\chi^{2}=7.8$ and 8.0. The fit for the Ge(111)5 $\times 5-\mathrm{Sn}$ data gave $\chi^{2}=10.7$

The electron density is proportional to the Fourier sum of the structure factors. The amplitudes of the structure factors are known both from experiment and for the model. When the phases of the model structure factors 
are assigned to both experimental and the model structure factors, the Fourier sum of the difference between them gives an estimate of the difference in the electron density. After each fit such an error synthesis was performed. Figure 5 shows plots of the positive contours in the electron density difference $\Delta \rho$ for the $7 \times 7$ data set 2 and for the $5 \times 5$ data set. The figure shows that the model should have more electron density at the positions of the adatoms. The error synthesis for data set 1 was similar.

Guided by the error synthesis, the electron density of the adatoms was allowed to vary in the next models. This was done by assuming the adatoms to be $\mathrm{Sn}$ and multiplying the form factor $f_{\mathrm{Sn}}$ by a fitting parameter. The form factors are proportional to the atomic charge $Z$ and have almost the same dependence on the scattering vector $Q$. So scaling the form factor of the adatoms is equivalent to fitting the charge of the adatoms. In the model we used one DW factor for the adatoms and another for the $\mathrm{Ge}$ atoms and the atoms were allowed to relax according to $6 \mathrm{~mm}$ symmetry. For the $7 \times 7$ data sets it was not possible to separate the displacements of the adatoms and the atoms just below them. These atoms have nearly coincident in-plane positions and cannot be distinguished in the fits of the in-plane projected models. Therefore, the atoms below the adatoms were fixed at their ideal positions. The results of the fitting procedure are displayed in Table II together with the results for $\operatorname{Si}(111) 7 \times 7$ by Robinson et al. ${ }^{11}$ (a) Ge(111)7×7-Sn

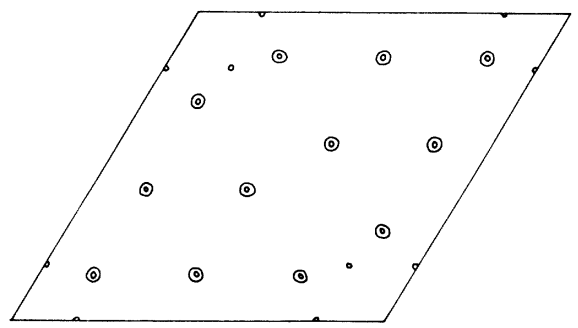

(b) Ge(111) $5 \times 5-\mathrm{Sn}$

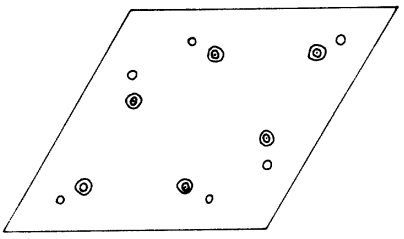

FIG. 5. Plots of the positive contours of the electron density difference $\Delta \rho$ for (a) the $7 \times 7$ data set 2 and (b) the $5 \times 5$ data set.

The least-squares fits of the models have values of $\chi^{2}$ which are more than a factor of 2 lower than the starting models. A comparison of the atomic displacements in Table II shows that the three structures, Ge(111)5 $\times 5-\mathrm{Sn}$,

TABLE II. The results from the fits of the $6 \mathrm{~mm}$ DAS model. The labels of the atoms refers to Fig. 4. The absolute displacements are given by multiplying by $a=4.000 \AA$ for the Ge structures and $a=3.840 \AA$ for the Si structure. The results for the $\mathrm{Si}(111) 7 \times 7$ surface are from Robinson et al. (Ref. 11). The form factors $f_{\text {ad }}$ for the adatoms and the $B$ factors are also displayed. The numbers in parentheses after the displacements are the uncertainties on the last digits. An asterisk indicates a parameter fixed by symmetry. Two asterisks indicate parameters that have been fixed in the least-squares fit.

\begin{tabular}{|c|c|c|c|c|c|}
\hline \multirow[b]{3}{*}{ Atom } & \multirow[b]{3}{*}{$\begin{array}{l}\text { Displacement } \\
\text { vector }\end{array}$} & \multicolumn{4}{|c|}{$\mathrm{Ge}(111) 7 \times 7-\mathrm{Sn}$} \\
\hline & & \multirow{2}{*}{$\begin{array}{c}\mathrm{Ge}(111) 5 \times 5-\mathrm{Sn} \\
\chi^{2}=5.1\end{array}$} & \multirow[b]{2}{*}{$\begin{array}{c}\text { No. } 1 \\
\chi^{2}=2.7\end{array}$} & \multirow[b]{2}{*}{$\begin{array}{c}\text { No. } 2 \\
\chi^{2}=3.6\end{array}$} & \multirow{2}{*}{$\begin{array}{c}\operatorname{Si}(111) 7 \times 7 \\
\chi^{2}=1.9\end{array}$} \\
\hline & & & & & \\
\hline 1 & $1 / \sqrt{3}(2,1)$ & $0.031(6)$ & $0.017(5)$ & $0.020(4)$ & $-0.007(3)$ \\
\hline 11 & $1 / \sqrt{3}(2,1)$ & $-0.018(10)$ & $0.0^{* *}$ & $0.0^{* *}$ & $-0.007(3)$ \\
\hline 2 & $1 / \sqrt{3}(1, \overline{1})$ & & $0.026(5)$ & $0.018(4)$ & $-0.005(3)$ \\
\hline 13 & $1 / \sqrt{3}(1, \overline{1})$ & & $0.0^{* *}$ & $0.0^{* *}$ & $-0.005(3)$ \\
\hline 3 & $1 / \sqrt{3}(2,1)$ & $0.028(4)$ & $0.025(7)$ & $0.041(6)$ & $0.031(9)$ \\
\hline 4 & $(1,1), 1 \sqrt{3}(1, \overline{1})$ & $-0.023(3),-0.001(3)$ & $-0.029(6),-0.013(5)$ & $-0.025(4),-0.012(4)$ & $-0.029(8), 0.007(12)$ \\
\hline 5 & $(1,1), 1 / \sqrt{3}(1, \overline{1})$ & & $0.010(4), 0.004(3)$ & $0.028(4),-0.002(4)$ & $0.030(7),-0.003(12)$ \\
\hline 6 & $1 / \sqrt{3}(2,1)$ & $0.0^{*}$ & $-0.041(7)$ & $-0.006(6)$ & $-0.016(9)$ \\
\hline 7 & $1 / \sqrt{3}(1, \overline{1})$ & & $0.005(7)$ & $0.000(5)$ & $-0.033(9)$ \\
\hline 8 & $(1,1)$ & $0.163(4)$ & $0.179(7)$ & $0.147(5)$ & $0.151(8)$ \\
\hline 9 & $(1,1)$ & $-0.180(4)$ & $-0.176(7)$ & $-0.172(5)$ & $-0.195(8)$ \\
\hline 10 & $(1,1)$ & & $0.179(7)$ & $0.175(5)$ & $0.178(8)$ \\
\hline 12 & $(1,1), 1 / \sqrt{3}(1, \overline{1})$ & $0.0^{*},-0.004(5)$ & $-0.011(5), 0.001(5)$ & $-0.011(4), 0.004(4)$ & $-0.015(6),-0.014(10)$ \\
\hline \multirow[t]{2}{*}{14} & $1 / \sqrt{3}(2,1)$ & & $-0.035(7)$ & $-0.043(6)$ & $-0.007(7)$ \\
\hline & & $\begin{array}{c}B_{\mathrm{ad}}=1.6 \pm 0.7 \AA^{2} \\
B_{\mathrm{Ge}}=1.7 \pm 0.3 \AA^{2} \\
f_{\mathrm{ad}}=0.86 \pm 0.05 f_{\mathrm{Sn}}\end{array}$ & $\begin{array}{c}B_{\mathrm{ad}}=0.2 \pm 0.6 \AA^{2} \\
B_{\mathrm{Ge}}=2.5 \pm 0.6 \AA^{2} \\
f_{\mathrm{ad}}=0.81 \pm 0.04 f_{\mathrm{Sn}}\end{array}$ & $\begin{array}{c}B_{\mathrm{ad}}=2.0 \pm 0.3 \AA^{2} \\
B_{\mathrm{Ge}}=1.2 \pm 0.2 \AA^{2} \\
f_{\mathrm{ad}}=0.97 \pm 0.04 f_{\mathrm{Sn}}\end{array}$ & $\begin{aligned} B_{\mathrm{ad}} & =1.5 \pm 0.6 \AA^{2} \\
B_{3-7} & =1.5 \pm 0.6 \AA^{2} \\
B_{8-14} & =0.0 \pm 0.4 \AA^{2}\end{aligned}$ \\
\hline
\end{tabular}


$\mathrm{Ge}(111) 7 \times 7-\mathrm{Sn}$, and $\mathrm{Si}(111) 7 \times 7$, have nearly the same atomic displacements. The reproducibility of the two independent sets of data from the $\mathrm{Ge}(111) 7 \times 7-\mathrm{Sn}$ samples is most satisfactory. As indicated by the error synthesis performed on the starting models, the adatoms are found to have more charge than the deeper atoms. For the $7 \times 7$ data set 2 the adatom charge corresponds to that of Sn. For the other data sets the charges at the adatom sites are smaller. However, the analysis does not give the absolute charge, but only the charge relative to the atoms in the deeper layers. The observed reduction of the adatom charge relative to a full occupation of the adatom sites by $\mathrm{Sn}$ can be caused by the following effects: (1) missing adatoms, (2) some Ge adatoms, (3) Sn substitution in the layers below the adatoms. The result for data set 2 can only occur when no Sn substitution is present in the layers below the adatoms and when all adatom positions are occupied by $\mathrm{Sn}$ atom. For data set 1 the adatom charge corresponds to an $81 \pm 4 \%$ occupation of the adatoms sites by $\mathrm{Sn}$, assuming that the deeper atoms are Ge. However, the $B$ factors or equivalently the vibration amplitude for the adatom is unrealistically small compared with those of the deeper atoms. This is probably due to the correlation in the model between the adatom charge and the $B$ factor. A first-order expansion of the DebyeWaller factor gives $\exp \left[-B Q^{2} /(4 \pi)^{2}\right]=1-B Q^{2} /(4 \pi)^{2}$. Data set 1 contains a limited number of reflections with relatively small scattering vectors $Q$ so the adatom charge is correlated with the $B$ factor. The occupation of $81 \pm 4 \%$ can be considered as a lower estimate for this sample. The data set for the $5 \times 5$ reconstruction gives an adatom charge, corresponding to a Sn occupation of $86 \pm 5 \%$ of the adatom sites, under the assumption that the atoms in the layers below the adatoms are Ge.

Figures 6(a) and 7(a) show a graphical presentation of the atomic displacements for the $\mathrm{Ge}(111) 7 \times 7-\mathrm{Sn}$ data set 2 and the $\mathrm{Ge}(111) 5 \times 5-\mathrm{Sn}$ data set, respectively. The displacements are shown as arrows and have been multiplied by 10 except for the dimer atoms, which are shown at their actual positions. The main features in the displacement patterns are net displacements of the atoms bonded to the adatoms towards them. For the $7 \times 7$ reconstruction it is $0.016 \pm 0.005$ for the adatom labeled 1 in Fig. 4 (a) and $0.014 \pm 0.005$ for the adatom labeled 2. The displacements quoted in this paper are all in units of the lat-

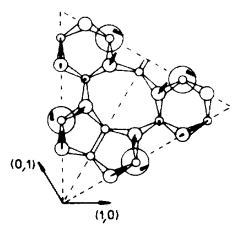

(a)

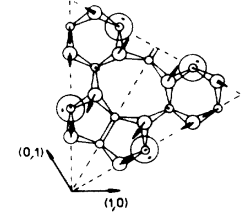

(b)

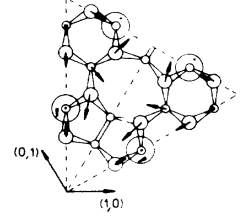

(c)
FIG. 6. The atomic displacements of the Ge(111)7 $\times 7-\mathrm{Sn}$ structure. The dimer atoms are shown at their actual positions and for the other atoms the arrows show the displacement multiplied by 10. (a) The result of the x-ray scattering experiment as shown in Tables II and IV. (b) From tight-binding calculations for $\mathrm{Si}(111) 7 \times 7$ (Ref. 10). (c) From a Keating calculation for $\mathrm{Ge}(111) 7 \times 7-\mathrm{Sn}$.

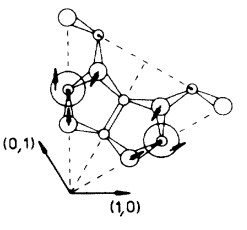

(a)

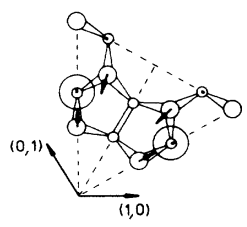

(b)

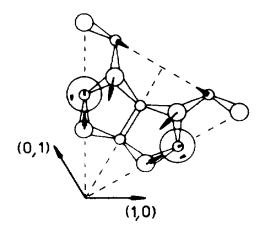

(c)
FIG. 7. The atomic displacements for the $\mathrm{Ge}(111) 5 \times 5-\mathrm{Sn}$ structure. The dimer atoms are shown at their actual position and for the other atoms the arrows show the displacements multiplied by 10 . (a) The results of the $x$-ray scattering experiment as shown in Tables II and V. (b) From the tight-binding calculations for the $\mathrm{Si}(111) 5 \times 5$ structure (Ref. 10). (c) From the Keating calculation for the Ge(111)5 $\times 5$-Sn surface.

tice constant of the $1 \times 1$ surface: $a=4.000 \AA$ for $\mathrm{Ge}$ and $a=3.840 \AA$ for $\mathrm{Si}$. The net displacements for the $5 \times 5$ structure of the atoms bonded to the adatoms is $0.021 \pm 0.004$, which agrees quite well with the displacements for the $7 \times 7$ structure. For comparison the $\mathrm{Si}(111) 7 \times 7$ has a net displacement of $0.029 \pm 0.005$ around both adatoms ${ }^{11}$ and the Ge(111) $\sqrt{3} \times \sqrt{3}-\mathrm{Sn}$ (Ref. 15) structure has a displacement of $0.051 \pm 0.004$.

The projected distance between the two atoms of the dimers are, for the $\mathrm{Ge}(111) 7 \times 7-\mathrm{Sn}$ data set 2, $0.678 \pm 0.007$ (between atoms 8 and 9), and $0.650 \pm 0.007$ (between atom 10 and its mirror image) compared to the bulk bond length of 0.613 . For the ideal unrelaxed model the dimer bonds are parallel to the surface. If they are assumed to be parallel to the surface also for the relaxed structures, the observed bond lengths correspond to a stretching of $11 \pm 1 \%$ and $6 \pm 1 \%$, respectively, for the two bonds. The $5 \times 5$ structure has a dimer bond length of $0.657 \pm 0.007$ with corresponds to a $7 \pm 1 \%$ stretch. For the $\mathrm{Si}(111) 7 \times 7$ structure $^{11}$ the average stretching of the dimers is $6 \pm 2 \%$. The atomic displacements are discussed further in the next section, where they are compared to the displacements obtained from theoretical calculations.

The agreements of the least-squares fits of the models with the $5 \times 5$ data set and the $7 \times 7$ data set 2 are $\chi^{2}=5.1$ and 3.6, which still leaves room for improvement. The $5 \times 5$ model requires 13 parameters and the $7 \times 7$ requires 19 , whereas the data sets contain, respectively, 115 and 269 reflections. In crystallography it is customary that about five reflections should be available for each parameter to be refined. Even applying this criterion the data sets are sufficiently large that more parameters can be included in the models. An obvious way to proceed is to allow the atoms to relax according to the lower $3 \mathrm{~m}$ symmetry of the bulk. The further analysis is restricted to the $5 \times 5$ data set and the $7 \times 7$ data set 2 , because only these two data sets have a sufficiently large number of reflections.

The next model that was fitted to the data included the adatoms and the next two bilayers. The adatoms and the first bilayer were relaxed according to $6 \mathrm{~mm}$ symmetry in order to limit the number of fitting parameters. The choice of $6 \mathrm{~mm}$ symmetry for these atoms corresponds to assuming that the displacements in the two parts of the 
unit cell with regular and faulted stacking are the same. The interaction that gives an energy difference between the two parts of the unit cell is between the fourthnearest-neighbor atoms and can be expected to have only a small influence on the nearest-neighbor bonds at the surface. The energy associated with a stacking fault in the surface layers has been calculated by Vanderbilt. ${ }^{16}$ The $a b$ initio total-energy calculations showed that for the relaxed $\mathrm{Si}(111) 1 \times 1$ surface, the energy difference between the regular and faulted surface is $60 \mathrm{meV}$ per $1 \times 1$ surface unit cell. For the $2 \times 2$ adatom model the energy difference is only $20 \mathrm{meV}$ per $1 \times 1$ area. These results agree with the energy of the bulk stacking fault in Si of about $30 \mathrm{meV}$ per $1 \times 1$ surface area ${ }^{17}$ and the energy difference between the cubic and hexagonal diamond structure of $32 \mathrm{meV}$ per $1 \times 1$ surface area for $\mathrm{Si}$ and 30 meV per $1 \times 1$ surface area Ge. ${ }^{18}$ The energy estimated for the stacking fault is negligible compared with the energy of a nearest-neighbor bond, which is of the order of $3 \mathrm{eV}^{19}$

The atoms in the second bilayer, which has $3 m$ symmetry, can be expected to have significant displacements. For the $\mathrm{Ge}(111) \sqrt{3} \times \sqrt{3}-M(M=\mathrm{Sn}$ or $\mathrm{Pb})$ structure which has a similar adatom geometry, ${ }^{15}$ the displace-

TABLE III. Atomic displacements of the atoms in the first two bilayers of the $7 \times 7$ and $5 \times 5$ DAS models. The atom labeling refers to Fig. 4. The models that have been used to obtain the experimental displacements are described in the caption of Fig. 4 and in the text. The parameters marked with an asterisk have been fixed in the least-squares fits. The displacements in the other columns have been found by a minimization of the elastic strain energy (Keating calculation).

\begin{tabular}{|c|c|c|c|c|c|}
\hline \multirow[b]{2}{*}{$\begin{array}{l}\text { Atom } \\
\text { no. }\end{array}$} & \multirow[b]{2}{*}{$\begin{array}{l}\text { Displacement } \\
\text { vectors }\end{array}$} & \multicolumn{2}{|c|}{$\mathrm{Ge}(111) 7 \times 7-\mathrm{Sn}$} & \multicolumn{2}{|c|}{$\mathrm{Ge}(111) 5 \times 5-\mathrm{Sn}$} \\
\hline & & $\begin{array}{c}\text { Experiment } \\
\chi^{2}=2.4 \\
\end{array}$ & $\begin{array}{l}\text { Keating } \\
\text { calc. }\end{array}$ & $\begin{array}{c}\text { Experiment } \\
\chi^{2}=3.2\end{array}$ & $\begin{array}{c}\text { Keating } \\
\text { calc. }\end{array}$ \\
\hline 1 & $1 / \sqrt{3}(2,1)$ & $0.016(4)$ & -0.014 & $0.030(7)$ & -0.009 \\
\hline 11 & $1 / \sqrt{3}(2,1)$ & $0.0^{*}$ & 0.003 & $-0.013(10)$ & 0.007 \\
\hline 2 & $1 / \sqrt{3}(1, \overline{1})$ & $0.026(5)$ & -0.005 & & \\
\hline 13 & $1 / \sqrt{3}(1, \overline{1})$ & $0.0^{*}$ & 0.004 & & \\
\hline $\begin{array}{l}3 \\
4\end{array}$ & $\begin{array}{c}1 / \sqrt{3}(2,1) \\
(1,1), 1 / \sqrt{ } 3(1, \overline{1})\end{array}$ & $\begin{array}{c}0.036(6) \\
-0.022(8), 0.001(7)\end{array}$ & $\begin{array}{c}0.024 \\
-0.049,-0.016\end{array}$ & $\begin{array}{c}0.033(5) \\
-0.030(4),-0.007(5)\end{array}$ & $\begin{array}{c}0.028 \\
-0.042,-0.012\end{array}$ \\
\hline 5 & $(1,1), 1 / \sqrt{3}(1, \overline{1})$ & $0.025(5), 0.016(6)$ & $0.040,-0.009$ & & \\
\hline 6 & $1 / \sqrt{3}(2,1)$ & $-0.017(7)$ & 0.033 & & \\
\hline 7 & $1 / \sqrt{3}(1, \overline{1})$ & $-0.025(7)$ & -0.032 & & \\
\hline 8 & $(1,1)$ & $0.152(5)$ & 0.156 & $0.161(4)$ & 0.161 \\
\hline 9 & $(1,1)$ & $-0.179(5)$ & -0.213 & $-0.184(4)$ & -0.207 \\
\hline 10 & $(1,1)$ & $0.178(5)$ & 0.187 & & \\
\hline 12 & $(1,1), 1 / \sqrt{3}(1, \overline{1})$ & $0.003(5), 0.004(4)$ & $-0.008,-0.036$ &,$--0.010(4)$ &,--0.027 \\
\hline 14 & $1 / \sqrt{3}(2,1)$ & $-0.049(6)$ & -0.019 & & \\
\hline 15 & $(1,1), 1 / \sqrt{3}(1, \overline{1})$ & $-0.021(6), 0.017(10)$ & $0.011,0.001$ & $-0.007(4),-0.015(9)$ & $0.013,0.000$ \\
\hline 16 & $(1,1), 1 / \sqrt{3}(1, \overline{1})$ & $-0.008(5),-0.005(15)$ & $-0.018,0.000$ & $-0.012(4),-0.005(13)$ & $-0.016,0.000$ \\
\hline 17 & $(1,1), 1 / \sqrt{3}(1, \overline{1})$ & $0.006(6),-0.011(12)$ & $0.012,0.001$ & & \\
\hline 18 & $1 / \sqrt{3}(1, \overline{1})$ & $0.0^{*}$ & -0.001 & & \\
\hline 19 & $1 / \sqrt{3}(1,2)$ & $0.0^{*}$ & -0.005 & & \\
\hline 20 & $(1,1), 1 / \sqrt{3}(1, \overline{1})$ & $0.0^{*}, 0.0^{*}$ & $-0.002,0.006$ &,$- 0.0^{*}$ &,- 0.004 \\
\hline 21 & $1 / \sqrt{3}(1,2)$ & $0.0^{*}$ & 0.001 & $0.0^{*}$ & 0.002 \\
\hline \multicolumn{6}{|c|}{ 然 } \\
\hline 23 & $1 / \sqrt{3}(2,1)$ & $0.0^{*}$ & 0.000 & $0.0^{*}$ & 0.000 \\
\hline 24 & $(1,1), 1 / \sqrt{3}(1, \overline{1})$ & $0.0^{*}, 0.0^{*}$ & $-0.002,0.004$ &,$- 0.0^{*}$ &,--0.005 \\
\hline 25 & $1 / \sqrt{3}(1, \overline{1})$ & $0.0^{*}$ & -0.001 & & \\
\hline 26 & $1 / \sqrt{3}(2,1)$ & $0.0^{*}$ & -0.003 & & \\
\hline \multicolumn{6}{|l|}{27} \\
\hline 28 & $(1,1), 1 / \sqrt{3}(1, \overline{1})$ & $-0.033(8), 0.002(8)$ & $-0.015,-0.004$ & & \\
\hline 29 & $1 / \sqrt{3}(1, \overline{1})$ & $-0.053(9)$ & -0.009 & & \\
\hline 30 & $(1,1), 1 / \sqrt{\overline{3}}(1, \overline{1})$ & $-0.009(8),-0.015(7)$ & $-0.004,-0.006$ &,$--0.020(8)$ &,--0.007 \\
\hline 31 & $(1,1), 1 / \sqrt{3}(1, \overline{1})$ & $-0.007(10), 0.023(10)$ & $-0.007,0.005$ & & \\
\hline 32 & $1 / \sqrt{3}(1, \overline{1})$ & $-0.030(14)$ & -0.015 & & \\
\hline 33 & $1 / \sqrt{3}(1,2)$ & $-0.036(8)$ & -0.007 & & \\
\hline 34 & $(1,1), 1 / \sqrt{3}(1, \overline{1})$ & $-0.014(15), 0.026(14)$ & $0.011,0.008$ & $0.020(7), 0.000(9)$ & $0.014,0.007$ \\
\hline 35 & $1 / \sqrt{3}(1,2)$ & $-0.046(10)$ & -0.012 & $-0.012(10)$ & -0.011 \\
\hline 36 & $1 / \sqrt{3}(2,1)$ & $-0.049(11)$ & 0.005 & $-0.044(7)$ & 0.009 \\
\hline 37 & $(1,1), 1 / \sqrt{3}(1, \overline{1})$ & $0.004(7),-0.014(6)$ & $-0.001,-0.009$ & $-0.006(5),-0.015(5)$ & $0.001,-0.009$ \\
\hline 38 & $1 / \sqrt{3}(2,1)$ & $0.032(10)$ & 0.016 & $0.021(6)$ & 0.016 \\
\hline
\end{tabular}


ments in the second bilayer are half as large as the displacements in the first bilayer. In the least-squares fits of the model, it is not possible to determine the relaxations of the atoms in the second bilayer that have atoms just above them. Figure 4 shows the labeling of the atoms in the models. For the $7 \times 7$ model the atoms labeled 18-26 were fixed and for the $5 \times 5$ model the atoms $20,21,23$, and 24 were fixed. The models have now 40 parameters for the $7 \times 7$ and 22 for the $5 \times 5$ structure. The leastsquares fits of these models gave the residuals $\chi^{2}=2.4$ and 3.2 for the $7 \times 7$ and $5 \times 5$ structures, respectively, compared to 3.6 and 5.1 for the previous models including only the adatoms and the first bilayer. The atomic displacements were displayed in Table III and are shown graphically in the left-hand sides of the Figs. 8 and 9. As before, the dimer atoms are shown at their actual positions, and for the other atoms the arrows show the displacement multiplied by 10 . The shaded atoms have been fixed in the fits.

The displacements of the adatoms and the atoms in the bilayer just below them are nearly the same as for the model without the second bilayer. The dominant feature in the displacement patterns is the displacement of the atoms bonded to the adatoms towards them. For the $7 \times 7$ data, the net displacements of the atoms bonded to the adatoms towards them have changed to $0.025 \pm 0.005$ and $0.038 \pm 0.005$ around adatoms 1 and 2, respectively. For the $5 \times 5$ data the displacement is $0.021 \pm 0.004$. These values are close to the displacement of the $\mathrm{Si}(111) 7 \times 7$ structure (Ref. 11): $0.029 \pm 0.005$, but smaller than the value for the $\alpha$ phase of the Ge(111) $\sqrt{3} \times \sqrt{3}-\mathrm{Sn}$ structure (Ref. 15): $0.051 \pm 0.004$. The bond length of (a)

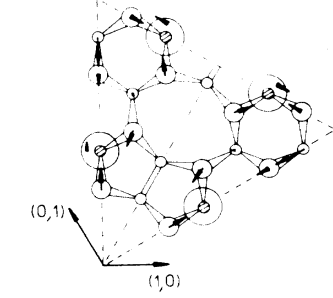

(b)

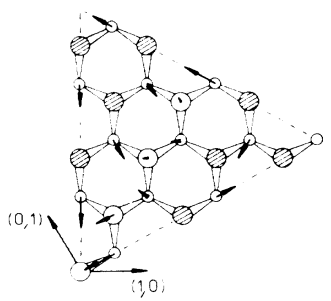

(c)

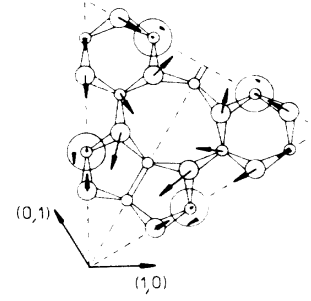

(d)

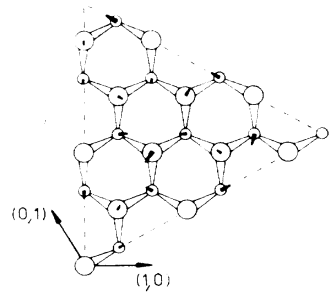

FIG. 8. The displacements of the atoms in the first two bilayer of the DAS model for the Ge(111)7 $\times 7$-Sn structure. The dimer atoms are at their actual positions and the displacements of the other atoms, multiplied by ten, are shown by arrows. Left-hand side: Experimental displacements. (a) Adatoms and first bilayer. (b) Second bilayer. The shaded atoms have been fixed in the least-squares fit. Right-hand side: Displacements from the Keating calculation. (c) Adatoms and first bilayer, (d) second bilayer. the dimers are nominally the same as for the previous models, which only included adatoms and the first bilayer.

The displacements of the second-layer atoms are mainly displacements of the fourth-layer atoms radially away from the projected positions of the adatoms. Hence, the displacement patterns around the adatoms in the $7 \times 7$ and $5 \times 5$ structures are similar to the displacement pattern of the Ge(111) $\sqrt{3} \times \sqrt{3}-\mathrm{Sn}$ surface. ${ }^{15}$ The adatoms are bonded to the first-layer atoms and pull these atoms towards them, The second- and third-layer atoms just below the adatoms are pushed in the direction normal to the surface into the crystal. This pushes the fourth-layer atoms bonded to the third-layer atoms away from the projected position of the adatom. For the $\mathrm{Ge}(111) 7 \times 7$ Sn structure the average in-plane displacement of the fourth-layer atoms is $0.038 \pm 0.005$ and for the $\mathrm{Ge}(111) 5 \times 5-\mathrm{Sn}$ structure it is $0.027 \pm 0.005$. These values are similar to the value for the $\operatorname{Ge}(111) \sqrt{3} \times \sqrt{3}$ Sn surface (Ref. 15): $0.027 \pm 0.003$. However, it should be noted that the displacements in the second bilayer are as large as the displacements in the first bilayer.

An error synthesis was performed after the leastsquares fits. For the $7 \times 7$ data set the contour plot of the electron density difference was flat and noisy. In contrast, the contour plot for the $5 \times 5$ structure showed additional electronic charge on the atom with the dangling bond, labeled 6 in Fig. 4(c), on the dimer atom labeled 8 closest to the corner holes, and in a position close to the dangling bond on atom 22 in the corner hole. In the next least-squares fit these electron charges were allowed to vary. This model gave a $\chi^{2}=1.7$ for the following electron charges: At atom $61.08 \pm 0.07$ and on the dimer atom $81.21 \pm 0.06$, both in units of the electronic charge of a $\mathrm{Ge}$ atom. The electronic charge in the corner hole was described by including one atom in each corner hole. The atom is displaced away from the atom with a dangling bond in the center of the corner hole. It can sit at (a)

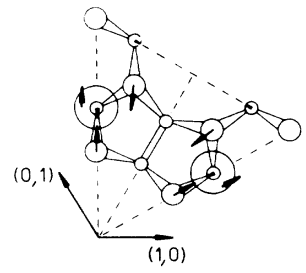

(b)

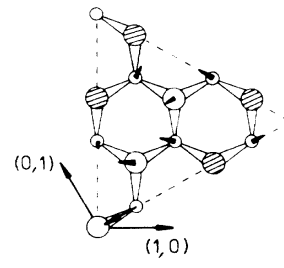

(c)

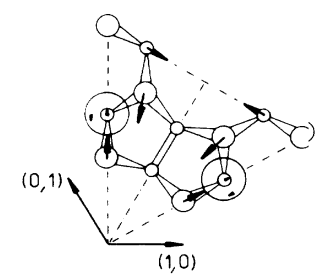

(d)

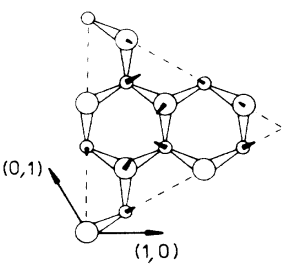

FIG. 9. The displacements of the Ge(111)5 $\times 5$-Sn structure. Left-hand side: experiment. Right-hand side: from an elastic strain energy minimization. See Fig. 8 for additional information. 
three different symmetry-equivalent positions. However, the electronic density corresponds to a situation in which the three positions are $33.3 \%$ occupied by $\mathrm{Sn}$ atoms, i.e., for each unit cell only one of the three positions is occupied. The atom has a $B$ factor of $5.4 \pm 1.5 \AA$ and is situated at the position $(0.18 \pm 0.03,0.35 \pm 0.06)$ or at one of the two equivalent positions. This situation cannot be distinguished from an occupation of all three positions with atoms having a charge of $\frac{1}{3}$ of an Sn atom. In this final model the adatom charge corresponds to $0.90 \pm 0.05$ of the electron charge of a $\mathrm{Sn}$ atom.

Atom 6 has only a marginally larger electronic charge than a $\mathrm{Ge}$ atom and so no further interpretation is possible. However, the additional charge on the dimer is significant and can be due to Sn substitution at this position. Assuming that the nonadatom positions are occupied by $\mathrm{Ge}$ atoms the $\mathrm{Sn}$ substitution of the dimer atom is $37 \pm 11 \%$. This corresponds to about two Sn atoms per $5 \times 5$ unit cell. The implication of this is discussed in Sec. $\mathrm{V}$ which contains a discussion of the total energies of the structures.

\section{COMPARISONS WITH ATOMIC RELAXATIONS FROM MODEL CALCULATIONS}

The previous section demonstrated similar atomic displacements for the $\mathrm{Ge}(111) 7 \times 7-\mathrm{Sn}, \mathrm{Ge}(111) 5 \times 5-\mathrm{Sn}$, $\mathrm{Si}(111) 7 \times 7$, and $\mathrm{Ge}(111) \sqrt{3} \times \sqrt{3}-\mathrm{Sn}$ structures. In order to obtain a good basis for a further discussion of these similarities, the atomic coordinates can be compared to the results of model calculations. The preferred techniques for such calculations are $a b$ initio total-energy methods, but unfortunately, the large unit cell of the
DAS structures makes such calculations impossible. However, semiempirical tight-binding calculations have been performed for the $\mathrm{Si}(111) 5 \times 5$ and $\mathrm{Si}(111) 7 \times 7 \mathrm{DAS}$ structures by Qian and Chadi. ${ }^{10}$ In these calculations the positions of the adatoms and the atoms in the next three layers of the crystal have been optimized to give the lowest value for the total energy. The in-plane projection of the displacements are close to having $6 \mathrm{~mm}$ symmetry. The displacements in the two halves of the unit cell typically agree within $10 \%$. The average displacements of the two halves of the unit cells are displayed in Tables IV and $\mathrm{V}$.

The atomic relaxations can also be obtained by calculating and minimizing the elastic strain in the reconstructions. We have chosen the Keating model, ${ }^{9}$ which has been very successful in describing the elastic properties of covalently bonded crystals. In this model the elastic energy is described by a bond-stretching and a bondbending term and the equilibrium bond configuration is assumed to be tetrahedral. The model is purely classical and does not directly take changes in electronic structure into account. In order to perform calculations on surfaces with more than one kind of atom, a generalized form of the Keating model $^{9}$ was used:

$$
\begin{aligned}
E= & \alpha \sum_{\substack{\text { all } \\
\text { bonds }}}\left[\mathbf{x}_{i j}^{2}-\left(b_{i}+b_{j}\right)^{2}\right]^{2} \\
& +\beta \sum_{\substack{\text { all } \\
\text { bond } \\
\text { angles }}}\left[\mathbf{x}_{i j} \cdot \mathbf{x}_{i k}+\frac{1}{3}\left(b_{i}+b_{j}\right)\left(b_{i}+b_{k}\right)\right]^{2},
\end{aligned}
$$

where $\alpha$ and $\beta$ are the bond-stretching and bond-bending parameters, respectively. $\mathbf{x}_{i j}$ is the vector from atom $i$ to

\begin{tabular}{|c|c|c|c|c|c|c|}
\hline \multirow[b]{2}{*}{$\begin{array}{l}\text { Atom } \\
\text { no. }\end{array}$} & \multirow[b]{2}{*}{$\begin{array}{l}\text { Displacement } \\
\text { vector }\end{array}$} & \multicolumn{3}{|c|}{$\operatorname{Si}(111) 7 \times 7$} & \multicolumn{2}{|c|}{$\mathrm{Ge}(111) 7 \times 7-\mathrm{Sn}$} \\
\hline & & $\begin{array}{c}\text { Experiment }^{\mathrm{a}} \\
\chi^{2}=1.9\end{array}$ & $\begin{array}{c}\text { Tight- } \\
\text { binding } \\
\text { calculation }^{\mathrm{b}}\end{array}$ & $\begin{array}{c}\text { Keating } \\
\text { calculation }\end{array}$ & $\begin{array}{l}\text { Keating } \\
\text { calculation }^{\mathrm{c}}\end{array}$ & $\begin{array}{c}\text { Experiment }^{\mathrm{d}} \\
\chi^{2}=3.6\end{array}$ \\
\hline 1 & $1 / \sqrt{3}(2,1)$ & $-0.007(3)$ & -0.003 & -0.012 & -0.014 & $0.020(4)$ \\
\hline 11 & $1 / \sqrt{3}(2,1)$ & $-0.007(3)$ & 0.005 & 0.006 & 0.003 & $0.0^{*}$ \\
\hline 2 & $1 / \sqrt{3}(1, \overline{1})$ & $-0.005(3)$ & 0.007 & -0.006 & 0.005 & $0.018(4)$ \\
\hline 13 & $1 / \sqrt{3}(1, \overline{1})$ & $-0.005(3)$ & 0.005 & 0.006 & 0.004 & $0.0^{*}$ \\
\hline 3 & $1 / \sqrt{3}(2,1)$ & $0.031(9)$ & 0.031 & 0.034 & 0.024 & $0.041(6)$ \\
\hline 4 & $(1,1), 1 / \sqrt{3}(1, \overline{1})$ & $-0.029(8), 0.007(12)$ & $-0.038,-0.005$ & $-0.054,-0.011$ & $-0.049,-0.016$ & $-0.025(4),-0.012(4)$ \\
\hline 5 & $(1,1), 1 / \sqrt{3}(1, \overline{1})$ & $0.030(7),-0.003(12)$ & $0.034,0.004$ & $0.046,-0.004$ & $0.040,-0.009$ & $0.028(4),-0.002(4)$ \\
\hline 6 & $1 / \sqrt{3}(2,1)$ & $-0.016(9)$ & -0.024 & -0.033 & -0.033 & $-0.006(6)$ \\
\hline 7 & $1 / \sqrt{3}(1, \overline{1})$ & $-0.033(9)$ & -0.021 & -0.039 & -0.032 & $0.000(5)$ \\
\hline 8 & $(1,1)$ & $0.151(8)$ & 0.163 & 0.158 & 0.156 & $0.147(5)$ \\
\hline 9 & $(1,1)$ & $-0.195(8)$ & -0.206 & -0.212 & -0.213 & $-0.172(5)$ \\
\hline 10 & $(1,1)$ & $0.178(8)$ & 0.186 & 0.187 & 0.187 & $0.175(5)$ \\
\hline 12 & $(1,1), 1 / \sqrt{3}(1, \overline{1})$ & $-0.015(6),-0.014(10)$ & $-0.007,-0.001$ & $-0.007,-0.038$ & $-0.008,-0.036$ & $-0.011(4), 0.004(4)$ \\
\hline 14 & $1 / \sqrt{3}(2,1)$ & $-0.007(7)$ & -0.036 & -0.017 & -0.019 & $-0.043(6)$ \\
\hline
\end{tabular}

TABLE IV. Atomic relaxations for the $\mathrm{Si}(111) 7 \times 7$ and $\mathrm{Ge}(111) 7 \times 7-\mathrm{Sn}$ surfaces.

${ }^{\mathrm{a}} \mathrm{X}$-ray diffraction experiment by Robinson et al. (Ref. 11). The structural model has $6 \mathrm{~mm}$ symmetry and includes only the adatoms and first bilayer.

${ }^{\mathrm{b}}$ Tight-binding calculation by Qian and Chadi (Ref. 10).

'Elastic strain calculations by the Keating model.

${ }^{\mathrm{d}} \mathrm{X}$-ray diffraction experiment. The same model as (a), except that the Ge atoms below the adatoms were fixed. 
TABLE V. Atomic displacements for the $\mathrm{Si}(111) 5 \times 5$ and $\mathrm{Ge}(111) 5 \times 5-\mathrm{Sn}$ surfaces.

\begin{tabular}{|c|c|c|c|c|c|}
\hline \multirow[b]{2}{*}{$\begin{array}{l}\text { Atom } \\
\text { no. }\end{array}$} & \multirow[b]{2}{*}{$\begin{array}{l}\text { Displacement } \\
\text { vector }\end{array}$} & \multicolumn{2}{|c|}{$\operatorname{Si}(111) 5 \times 5$} & \multicolumn{2}{|c|}{$\mathrm{Ge}(111) 5 \times 5-\mathrm{Sn}$} \\
\hline & & $\begin{array}{l}\text { Tight- } \\
\text { binding } \\
\text { calc. }^{\mathrm{a}}\end{array}$ & $\begin{array}{l}\text { Keating } \\
\text { calculation }^{\mathrm{b}}\end{array}$ & $\begin{array}{l}\text { Keating } \\
\text { calculation }^{\mathrm{b}}\end{array}$ & $\begin{array}{c}\text { Experiment }^{\mathrm{c}} \\
\chi^{2}=5.1\end{array}$ \\
\hline $\begin{array}{r}1 \\
11\end{array}$ & $\begin{array}{l}1 / \sqrt{3}(2,1) \\
1 / \sqrt{3}(2,1)\end{array}$ & $\begin{array}{c}-0.001 \\
0.007\end{array}$ & $\begin{array}{c}-0.006 \\
0.011\end{array}$ & $\begin{array}{c}-0.009 \\
0.007\end{array}$ & $\begin{array}{c}0.031(6) \\
-0.018(10)\end{array}$ \\
\hline $\begin{array}{l}3 \\
4 \\
6\end{array}$ & $\begin{array}{c}1 / \sqrt{3}(2,1) \\
(1,1), 1 / \sqrt{3}(1, \overline{1})\end{array}$ & $\begin{array}{c}0.032 \\
-0.034,-0.004\end{array}$ & $\begin{array}{c}0.038 \\
-0.048,-0.008\end{array}$ & $\begin{array}{c}0.028 \\
-0.042,-0.012\end{array}$ & $\begin{array}{c}0.028(4) \\
-0.023(3),-0.001(3)\end{array}$ \\
\hline $\begin{array}{r}8 \\
9 \\
12\end{array}$ & $\begin{array}{c}(1,1) \\
(1,1) \\
1 / \sqrt{3}(1, \overline{1})\end{array}$ & $\begin{array}{c}0.166 \\
-0.202 \\
0.004\end{array}$ & $\begin{array}{c}0.162 \\
-0.206 \\
-0.029\end{array}$ & $\begin{array}{c}0.161 \\
-0.207 \\
-0.027\end{array}$ & $\begin{array}{l}0.163(4) \\
-0.180(4) \\
-0.004(5)\end{array}$ \\
\hline
\end{tabular}

${ }^{a}$ Tight-binding calculation by Qian and Chadi (Ref. 10).

${ }^{b}$ Elastic strain calculations by the Keating model.

${ }^{c} \mathrm{X}$-ray diffraction experiment. The model has $6 \mathrm{~mm}$ symmetry and includes only the adatoms and the first bilayer.

atom $j$ and $b_{1}$ is the covalent radius of atom $i$. The structure of a surface reconstruction is determined by the ratio $\beta / \alpha$, which was set to 0.10 for both $\mathrm{Si}$ (Ref. 20) and $\mathrm{Ge}$ (Ref. 21) surfaces. $\alpha$ and $\beta$ are not known for bonds between $\mathrm{Sn}$ and $\mathrm{Ge}$ and they were set to have the same values as for the substrate bonds. It has recently been demonstrated $^{12,22}$ that this model is able to give relaxations that agree quite well with more advanced calculations and with experiment. More details about the calculations can be found in Ref. 22.

We have calculated the positions of the adatoms, and the atoms in the first three bilayers (i.e., six layers) for the $5 \times 5$ and $7 \times 7$ DAS models by minimizing the elastic strain using the Keating model. The calculations involve the optimization of 171 and 89 parameters for the $7 \times 7$ and $5 \times 5$ structures, respectively. As for the tightbinding calculations the displacements of the adatoms and of the atoms in the first bilayer are found to have nearly $6 \mathrm{~mm}$ symmetry. The results for the $\mathrm{Si}(111) 7 \times 7$, $\mathrm{Ge}(111) 7 \times 7-\mathrm{Sn}, \mathrm{Sn}(111) 5 \times 5$, and $\mathrm{Ge}(111) 5 \times 5-\mathrm{Sn}$ are shown in Tables IV and V. The agreement between the results from tight-binding calculations and the Keating calculations is impressive, considering the very different approaches. The largest differences are for the atoms bonded to the adatoms [atoms 4 and 5 for the $\operatorname{Si}(111) 7 \times 7$ structure and atom 4 for the $\operatorname{Si}(111) 5 \times 5$ structure]. The bonds of the adatoms are heavily distorted and in the Keating calculations they are treated as bulk bonds. This is probably a too simplistic approach to account for the large deviations from the tetrahedral configuration.

The experimental displacements determined by $x$-ray diffraction for the $\mathrm{Si}(111) 7 \times 7$ (Ref. 11) and Ge(111)7 $\times 7$-Sn structures are also displayed in Table IV. The results are from the least-squares fits of the models with $6 \mathrm{~mm}$ symmetry, which includes the adatoms and the atoms in the first bilayer. The experimental results for the $\mathrm{Si}(111) 7 \times 7$ and the $\mathrm{Ge}(111) 7 \times 7-\mathrm{Sn}$ surface agree quite well with the tight-binding calculations as well as with the Keating calculations. The displacement of atoms bonded to the adatoms are closest to the results of the tight-binding calculations. This is reasonable, due to the more realistic treatment of electronic properties in this type of calculations. Figures 6 and 7 show a graphical presentation of the displacements. The displacements are multiplied by 10 except for the dimer atoms. Figure 6 shows the results from experiment for $\mathrm{Ge}(111) 7 \times 7-\mathrm{Sn}$, the tight-binding results for $\mathrm{Si}(111) 7 \times 7$ (Ref. 10) and the results from the Keating calculation for the Ge(111)7 $\times 7$-Sn surface. The experimental results have a good qualitative agreement with both types of calculations. However, none of them are able to predict the experimentally observed displacements of the adatoms. The adatoms are found to be relaxed away from the dimers and the corner hole, while the calculations give the opposite direction.

The experimental results for the Ge(111)5 $\times 5$-Sn structure are in very good agreement with the tight-binding results for the $\mathrm{Si}(111) 5 \times 5$ structure $^{10}$ and the results from Keating calculation, as shown by Table $V$ and Fig. 7. But again both types of calculation fail to predict the observed displacement of the adatom. The projected dimer bond lengths are found to be about $3 \%$ longer than the bulk bond lengths from both tight-binding and Keating calculations. This value is somewhat small compared to the experimental values. For the $\mathrm{Si}(111) 7 \times 7$ structure the dimer bonds are $6 \pm 2 \%$ longer, ${ }^{11}$ for the $\mathrm{Ge}(111) 7 \times 7-\mathrm{Sn}$ structure they are $11 \pm 1 \%$ and $6 \pm 1 \%$ longer, and the dimer bond for the Ge(111)5 $\times 5-\mathrm{Sn}$ surface is $7 \pm 1 \%$ longer than the bulk value.

The Keating calculations also give the displacements of the atoms in the deeper layers. They can be compared to the experimental results for the models that include the adatoms, the atoms in the first bilayer with $6 \mathrm{~mm}$ symmetry, and the atoms in the second bilayer with $3 \mathrm{~m}$ symmetry. The results from experiment and from Keating calculations for the $\mathrm{Ge}(111) 7 \times 7-\mathrm{Sn}$ and $\mathrm{Ge}(111) 5 \times 5-\mathrm{Sn}$ structures are displayed in Table III and shown in Figs. 8 and 9. The displacements have a good agreement except for the second-layer atoms bonded to the "rest atom" in the corner hole (atom 22 in Fig. 4), which has a dangling 
bond. A close inspection of the displacements in the upper layers (Table III and Figs. 8 and 9) also shows that the displacements of the atoms bonded to atom 6 with a dangling bond are not in good agreement with the results from the Keating calculation.

$A b$ initio total-energy calculations for the $\mathrm{Si}(111) 2 \times 2$ $\mathrm{Si}$ adatom surface ${ }^{19}$ with adatoms in threefoldcoordinated sites on top of the second layer of $\mathrm{Si}$ atoms show that the dangling-bond state of the adatom is at a higher energy than the dangling-bond state of the rest atom. This leads to charge transfer from the adatom to the rest atom and rehybridization of the rest atom. The bonds of the rest atom to the substate atoms become more $p$-like, and this gives rise to an outwards displacement of the rest atoms. ${ }^{23}$ In the in-plane projection this is observed as a displacement of the atoms bonded to the rest atoms towards the rest atoms. The tight-binding calculations by Qian and Chadi ${ }^{10}$ take the rehybridization of the rest atoms into account and therefore the atomic relaxations found by these calculations are in better agreement with experiment (Tables IV and V).

In summary, the observed in-plane atomic displacements of the $\mathrm{Ge}(111) 7 \times 7-\mathrm{Sn}$ and $\mathrm{Ge}(111) 5 \times 5-\mathrm{Sn}$ structures are well described by both Keating and tightbinding calculations. The tight-binding calculations give a better description of the relaxations in the upper layers, due to the more realistic treatment of the atoms which have changes in electronic configurations. For the deeper atoms, which have positions that are only slightly different from the bulk positions, the results from the Keating calculations are in good agreement with experiment.

\section{DISCUSSION}

The broken ("dangling") bonds of an ideally terminated $\mathrm{Si}(111)$ or $\mathrm{Ge}(111)$ surface are energetically unfavorable. Therefore, the surface atoms rebond in order to reduce the number of dangling bonds. The rebonding results in a deformation of the bonds of the near-surface atoms away from their ideal configuration. This costs elastic energy and limits the degree of rebonding. In this section we will discuss the total energy of the surface. Due to the similarities of the reconstructions observed on the $\mathrm{Si}(111)$ and $\mathrm{Sn}$-covered $\mathrm{Ge}(111)$ surfaces we will consider both the $\mathrm{Si}$ and Ge surface.

Before discussing the results from total-energy calculations it is convenient to estimate the importance of the electronic and elastic contributions to the total energy of $\mathrm{Si}$ relative to Ge. The energy per unit cell, $E_{1 \times 1}$, of the ideally terminated surface is the electronic energy of a dangling bond relative to that of the saturated bond in the bulk crystal. This energy gives the scale for the electronic energy reduction due to dangling-bond annihilation. This energy has been calculated by $a b$ initio methods and the ratio between the result for Si (Ref. 19) and that for Ge (Ref. 24) is

$$
E_{1 \times 1}^{\mathrm{Si}} / E_{1 \times 1}^{\mathrm{Ge}}=1.63 \mathrm{eV} / 1.22 \mathrm{eV}=1.34 .
$$

For comparison, the ratio between the cohesive energies is

$$
E_{\mathrm{coh}}^{\mathrm{S}_{1}} / E_{\mathrm{coh}}^{\mathrm{Ge}}=4.84 \mathrm{eV} / 4.26 \mathrm{eV}=1.14
$$

from $a b$ initio calculations, ${ }^{18}$ and

$$
E_{\mathrm{coh}}^{\mathrm{S}_{\mathrm{i}}} / E_{\mathrm{coh}}^{\mathrm{Ge}}=4.63 \mathrm{eV} / 3.85 \mathrm{eV}=1.20
$$

from experiment. ${ }^{25}$ The elastic energy cost associated with static deformations can be described by the Keating model. ${ }^{9}$ In the phonon short-wavelength limit, which is relevant for surface relaxations, the ratio between the parameters describing the energy cost of bond stretching $(\alpha)$ and bond bending $(\beta)$ is $\beta / \alpha \simeq 0.10$ for both $\mathrm{Si}$ (Ref. 20) and Ge (Ref. 21). Therefore, the ratio between energy scales for the elastic energy can be calculated from the values of $\alpha=0.201 \mathrm{eV} / \AA^{4}$ for Si and $\alpha=0.161 \mathrm{eV} / \AA^{4}$ for Ge. Taking the dependence on the lattice constant $a_{0}$ into account, the ratio is

$$
E_{\text {elas }}^{\mathrm{S}_{1}} / E_{\text {elas }}^{\mathrm{Ge}}=\left(a_{0}^{\mathrm{S}_{1}}\right)^{4} \alpha^{\mathrm{S}_{1}} /\left(a_{0}^{\mathrm{Ge}}\right)^{4} \alpha^{\mathrm{Ge}}=1.06 .
$$

This shows that for Ge the elastic energy cost is more important relative to the electronic energy gain than it is for Si [Eq. (4)].

For the Si(111) surface the $7 \times 7$ DAS model describes the thermodynamically most stable structure. ${ }^{5}$ In this model the formation of the rows of dimers costs a considerable amount of elastic energy. ${ }^{26,27}$ However, the dimer rows effectively reduce the number of dangling bonds, and for Si the DAS model is favorable. For Ge the elastic energy required for dimer formation compared to the gain in electronic energy is unfavorable and the surface forms a simple $c(2 \times 8)$ adatom structure with the adatoms in a local $2 \times 2$ arrangement. ${ }^{3}$ The Sn adatoms lead to smaller subsurface relaxations, and this consequently favors a $7 \times 7$ DAS structure over the simple adatom structure.

For the $5 \times 5$ Sn-induced structure we have found a partial Sn substitution of the dimer atom closest to the corner hole. This further reduces the elastic energy for the dimers and thus favors the higher density of dimers in the $5 \times 5$ structure compared to the $7 \times 7$ structure. The above gives a simple qualitative explanation for the observed structural transformation: $c(2 \times 8) \rightarrow 7 \times 7$ DAS $\rightarrow 5 \times 5$ DAS for increasing $S n$ coverage.

Table VI contains a compilation of some of the results of total-energy calculations for $\mathrm{Ge}(111)$ and $\mathrm{Si}(111)$ surfaces. The results are from $a b$ initio pseudopotential calculations employing a local density approximation, except for the $5 \times 5$ and $7 \times 7$ structures which are from semiempirical tight-binding calculations. ${ }^{10}$ The energies $\Delta E$ are given relative to the ideally terminated $1 \times 1$ surface and they are per $1 \times 1$ unit cell area. For the surfaces which have been analyzed by different groups, a considerable scatter in the results is observed. This is partly due to different energy cutoffs in the calculations and partly due to allowing the atoms to relax to a different extent.

Allowing the $1 \times 1$ surface to relax gives an energy of $-0.07 \mathrm{eV}$ for $\mathrm{Ge}$ (Ref. 24) and of about $-0.16 \mathrm{eV}$ for $\mathrm{Si}^{16,19,28}$ The $\pi$-bonded chain model proposed by Pandey $^{29}$ is the accepted model ${ }^{30}$ for the structures on the cleaved surfaces and it has an energy (per $1 \times 1$ unit cell) $-0.34 \mathrm{eV}$ for Ge (Refs. 31 and 32) and of about -0.45 
TABLE VI. Total energies for different structures on the Ge(111) and $\mathrm{Si}(111)$ surfaces. The results are from $a b$ initio pseudopotential calculations employing the local density approximation, except for the $5 \times 5$ and $7 \times 7$ structures, which are from tight-binding calculations. The $2 \times 1 \pi$-bonded structure refers to Pandey's model (Ref. 29). The $2 \times 2$ structure is a simple adatom model with the adatoms on top of the second-layer substrate atoms $\left(T_{4}\right.$ site). The energies are in $\mathrm{eV}$ per $1 \times 1$ unit cell.

\begin{tabular}{lcccc}
\hline \multicolumn{1}{c}{ Structure } & \multicolumn{3}{c}{$\mathrm{Ge}(111)$} & \multicolumn{2}{c}{$\mathrm{Si}(111)$} \\
& $E(\mathrm{eV})$ & $\Delta E(\mathrm{eV})$ & $E(\mathrm{eV})$ & $\Delta E(\mathrm{eV})$ \\
\hline $1 \times 1$ unrelaxed & $1.22^{\mathrm{a}}$ & 0.0 & $1.63^{\mathrm{c}}$ & 0.0 \\
$1 \times 1$ relaxed & $1.15^{\mathrm{a}}$ & -0.07 & $1.50,1.45^{\mathrm{d}}$ & $-0.13,-0.18$ \\
$2 \times 1 \pi$-bonded & $0.88^{\mathrm{b}}$ & $\sim-0.34$ & $1.39,1.35^{\mathrm{e}}$ & $-0.36,-0.47$ \\
$2 \times 2\left(T_{4}\right)$ & $1.04^{\mathrm{a}}$ & -0.18 & $1.27,1.16^{\mathrm{f}}$ & $-0.24,-0.28$ \\
$3 \times 3$ DAS & $1.12^{\mathrm{a}}$ & -0.34 & & \\
$5 \times 5$ DAS & & & $1.235^{\mathrm{g}}$ & -0.395 \\
$7 \times 7$ DAS & & & $1.243^{\mathrm{g}}$ & -0.403 \\
\hline \hline
\end{tabular}

${ }^{\mathrm{a}}$ Reference 24.

${ }^{b}$ Estimated from Refs. 31 and 32.

Reference 19.

${ }^{\mathrm{d}}$ References 16,19 , and 28 .

${ }^{\mathrm{e}}$ References 16,33 , and 28.

${ }^{f}$ References 16 and 19.

${ }^{\mathrm{g}}$ Reference 10.

$\mathrm{eV}$ for $\mathrm{Si}^{16,33,28}$ Therefore, models for the stable structures have to have total energies which are lower than these values. The simple adatom structure on the $\mathrm{Ge}(111) c(2 \times 8)$ (Ref. 3) surface is a small modification of the $2 \times 2$ adatom structure with the adatoms on top of the second layer substrate atoms $\left(T_{4}\right.$ site). However, the $2 \times 2$ structure has an energy which is relatively high for both Ge (Ref. 24) and Si (Refs. 16 and 19) compared to the energy of the $\pi$-bonded chain models. A calculation for the full $c(2 \times 8)$ unit cell is not yet available.

$A b$ initio calculations for the $3 \times 3$ DAS structure on Ge (Ref. 24) and tight-binding calculations for the $5 \times 5$ and $7 \times 7$ DAS structures on Si (Ref. 10) gave energies of the same magnitude as the $\pi$-bonded chain model. For Si the energy difference between the $5 \times 5$ and $7 \times 7$ structures is only $8 \mathrm{meV}$ per $1 \times 1$ unit cell. For the $\mathrm{Ge}(111) 5 \times 5$-Sn structure we have found a partial Sn substitution of the dimer atoms near the corner holes. We have calculated the elastic energy reduction due to this substitution by the Keating model. For the elastic parameters of $\mathrm{Si}$ (Ref. 20) a 33.3\% substitution of the dimer atoms with a $15 \%$ larger radius (corresponding to Sn substitution in $\mathrm{Ge}$ ) gives a $20-\mathrm{meV}$ reduction of the elastic energy. This is enough to account for the change from $7 \times 7$ to $5 \times 5$ DAS structure observed on the Ge(111)-Sn surfaces.

\section{SUMMARY AND CONCLUSIONS}

The analysis of the surface $x$-ray diffraction data has shown that the $\mathrm{Ge}(111) 7 \times 7-\mathrm{Sn}$ and $\mathrm{Ge}(111) 5 \times 5-\mathrm{Sn}$ structures are described by the DAS model with $\mathrm{Sn}$ adatoms. The refined atomic positions of the reconstructions are in good agreement with tight-binding calculations for the Si DAS structures. The agreement with elastic energy minimization by the Keating model is slightly worse due to the inability of this model to treat atoms which undergo modification of their electronic structure. The displacements in the second bilayer are in good qualitative agreement with the predictions of the Keating model.

For the $5 \times 5$ structure the analysis gives indications of a $\mathrm{Sn}$ atom in the vicinity of a dangling bond in the corner hole and of more charge on the dimer atom closest to the corner hole. About every third dimer atom was estimated to be substituted by $\mathrm{Sn}$ for the particular sample we measured. An elastic energy calculation using the Keating model shows that $\mathrm{Sn}$ substitution gives a decrease in elastic energy, which explains the formation of the $5 \times 5$ structure instead of the $7 \times 7$ structure.

For the DAS structures the density of adatoms is 0.245 for the $7 \times 7$ and 0.240 for the $5 \times 5$ structure. As the $7 \times 7$ structure is observed after deposition of $0.3-0.5$ monolayers of $\mathrm{Sn},{ }^{7}$ the adatoms account for practically all of the $\mathrm{Sn}$ in this reconstruction. The $\mathrm{Sn}$ coverage of the $5 \times 5$ structure estimated from the analysis of 0.37 monolayers. Ichikawa and Ino $^{7}$ determined that a minimum coverage of about 0.7 monolayers is required to obtain the $5 \times 5$ structure with no admixture of the $7 \times 7$ structure. The Sn atoms which are not located in the analysis could be either randomly substituted in the surface layers or be in disordered regions of the surface. A random distribution in the surface layers is plausible since the larger covalent radius of $\mathrm{Sn}$ compared to $\mathrm{Ge}$ means that $\mathrm{Sn}$ substitution favors dimer formation.

\section{ACKNOWLEDGMENTS}

We wish to thank the staff of HASYLAB for their assistance and J. Als-Nielsen, J. Bohr, K. Kjær, D. Vanderbilt, C. Reiss, and I. K. Robinson for fruitful and stimulating discussions. We also thank I. K. Robinson, D. Vanderbilt, and G.-X. Qian for making unpublished work available. The work was supported by the Danish Natural Science Foundation and the German Federal Minister for Science and Technology under Project No. $05390 \mathrm{CAB}$. 
*Present address: Ris $\varnothing$ National Laboratory, DK-4000 Roskilde, Denmark.

${ }^{1}$ W. A. Harrison, Electronic Structure (Freeman, San Francisco, 1980).

${ }^{2}$ P. W. Palmberg and W. T. Peria, Surf. Sci. 6, 57 (1967).

${ }^{3}$ P. M. J. Marée, K. Nakagawa and J. F. van der Veen, Phys. Rev. B 38, 1585 (1988); R. Feidenhans'l, J. Skov Pedersen, J. Bohr, M. Nielsen, F. Grey, and R. L. Johnson, Phys. Rev. B (to be published).

${ }^{4}$ H. E. Farnsworth, R. E. Schlier, and J. A. Dillon, J. Phys. Chem. Solids 8, 116 (1959).

${ }^{5} \mathrm{~K}$. Takayanagi, Y. Tanishiro, S. Takahashi, and M. Takahashi, Surf. Sci. 164, 367 (1985).

${ }^{6}$ H. J. Grossmann, J. C. Bean, L. C. Feldman, E. G. McRae, and I. K. Robinson, Phys. Rev. Lett. 55, 1106 (1985).

${ }^{7}$ T. Ichikawa and S. Ino, Surf. Sci. 105, 395 (1985); Solid State Commun. 27, 483 (1978).

${ }^{8}$ J. Skov Pedersen, R. Feidenhans'l, M. Nielsen, K. Kjær, F. Grey, R. L. Johnson, and C. Reiss, The Structure of Surfaces II, edited by J. F. van der Veen and M. A. van Hove (Springer-Verlag, Berlin, 1987), p. 352.

${ }^{9}$ P. N. Keating, Phys. Rev. 145, 637 (1966).

${ }^{10}$ G.-X. Qian and D. J. Chadi, Phys. Rev. B 35, 1288 (1987); (unpublished).

${ }^{11}$ I. K. Robinson, W. K. Waskiewicz, P. H. Fuoss, and L. J. Norton, Phys. Rev. B 37, 4325 (1988).

12J. Skov Pedersen, Ph.D. thesis, Copenhagen University, 1988. Available as Report Ris $\varnothing-M-2713$ on request to Ris $\varnothing$ Library, Ris $\varnothing$ National Laboratory, DK-4000 Roskilde, Denmark.

${ }^{13}$ B. E. Warren, $X$-Ray Diffraction (Addition-Wesley, Reading, Mass., 1968).

${ }^{14}$ J. Bohr, R. Feidenhans'l, M. Nielsen, M. Toney, R. L. Johnson, and I. K. Robinson, Phys. Rev. Lett. 56, 2878 (1986).
${ }^{15}$ J. Skov Pedersen, R. Feidenhans'l, M. Nielsen, K. Kjær, F. Grey, and R. L. Johnson, Surf. Sci. 189/190, 1047 (1987).

${ }^{16}$ D. Vanderbilt, Phys. Rev. Lett. 59, 1456 (1987).

${ }^{17}$ M. Y. Chou, M. L. Cohen, and S. G. Louie, Phys. Rev. B 32, 7979 (1985).

${ }^{18}$ M. T. Yin and M. L. Cohen, Phys. Rev. B 26, 5668 (1982).

${ }^{19}$ J. E. Northrup, in Proceedings of the 18th International Conference on the Physics of Semiconductors, Stockholm, 1986, edited by O. Engström (World Scientific, Singapore, 1987), p. 61.

${ }^{20}$ G. A. Baraff, E. O. Kane, and M. Schlüter, Phys. Rev. B 21, 5662 (1980).

${ }^{21}$ A. Steif, S. C. Tiersten, and S. C. Ying, Phys. Rev. B 35, 857 (1987).

22J. Skov Pedersen, Surf. Sci. (to be published).

${ }^{23}$ M. Schlüter, J. R. Chelikowsky, S. G. Louie, and M. L. Cohen, Phys. Rev. B 12, 4200 (1975).

${ }^{24}$ M. C. Payne, J. Phys. C 20, L983 (1987).

${ }^{25}$ L. Brewer, Lawrence Berkeley Laboratories Report No. LBL-3720, 1975 (unpublished).

${ }^{26}$ G.-X. Qian and D. J. Chadi, J. Vac. Sci. Technol. A 5, 906 (1987).

${ }^{27}$ D. Vanderbilt, Phys. Rev. B 36, 6209 (1987).

${ }^{28}$ O. H. Nielsen, R. M. Martin, D. J. Chadi, and K. Kunc, J. Vac. Sci. Technol. B 1, 714 (1983).

${ }^{29}$ K. C. Pandey, Phys. Rev. Lett. 47, 1913 (1981).

${ }^{30}$ M. A. Olmstead, Surf. Sci. Rep. 6, 159 (1987).

${ }^{31}$ J. E. Northrup and M. L. Cohen, J. Vac. Sci. Technol. 21, 333 (1982).

32 J. E. Northrup and M. L. Cohen, Phys. Rev. B 27, 6553 (1983).

${ }^{33}$ J. E. Northrup and M. L. Cohen, Phys. Rev. Lett. 49, 1349 (1982). 


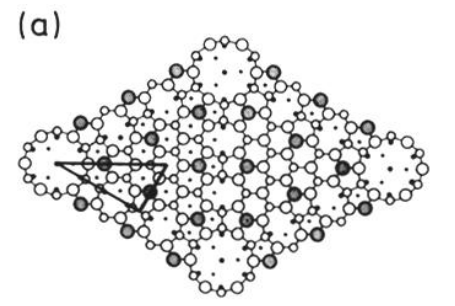

(b)

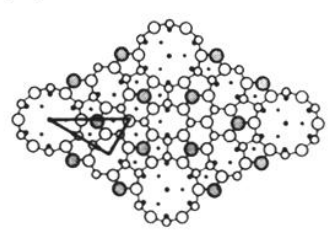

FIG. 3. The DAS model. (a) $7 \times 7$ and (b) $5 \times 5$. The adatoms are shaded and the irreducible unit of the Patterson function is shown by a triangle. 